\title{
Remarks on Star-Hurewicz Spaces
}

by

\author{
Yan-Kui SONG \\ Presented by Czesław BESSAGA
}

Summary. A space $X$ is star-Hurewicz if for each sequence $\left(\mathcal{U}_{n}: n \in \mathbb{N}\right)$ of open covers of $X$ there exists a sequence $\left(\mathcal{V}_{n}: n \in \mathbb{N}\right)$ such that for each $n, \mathcal{V}_{n}$ is a finite subset of $\mathcal{U}_{n}$, and for each $x \in X, x \in \operatorname{St}\left(\bigcup \mathcal{V}_{n}, \mathcal{U}_{n}\right)$ for all but finitely many $n$. We investigate the relationship between star-Hurewicz spaces and related spaces, and also study topological properties of star-Hurewicz spaces.

1. Introduction. By a space, we mean a topological space. Let $\mathbb{N}$ denote the set of positive integers. Let $X$ be a space and $\mathcal{U}$ be a collection of subsets of $X$. For $A \subseteq X$, let

$$
\operatorname{St}(A, \mathcal{U})=\bigcup\{U \in \mathcal{U}: U \cap A \neq \emptyset\} .
$$

As usual, we write $\operatorname{St}(x, \mathcal{U})$ instead of $\operatorname{St}(\{x\}, \mathcal{U})$.

Let us recall that a space $X$ is countably compact if every countable open cover of $X$ has a finite subcover. Van Douwen et al. [5] defined a space $X$ to be strongly starcompact if for every open cover $\mathcal{U}$ of $X$ there exists a finite subset $F$ of $X$ such that $\operatorname{St}(F, \mathcal{U})=X$. They proved that every countably compact space is strongly starcompact and every strongly starcompact $T_{2^{-}}$ space is countably compact; however, the latter does not hold for $T_{1}$-spaces (see [12, Example 2.5]).

Van Douwen et al. 5] defined a space $X$ to be starcompact if for every open cover $\mathcal{U}$ of $X$ there exists a finite subset $\mathcal{V}$ of $\mathcal{U}$ such that $\operatorname{St}(\bigcup \mathcal{V}, \mathcal{U})$ $=X$.

In [7], a strongly starcompact space is called starcompact, and in [11], a starcompact space is called $1 \frac{1}{2}$-starcompact.

2010 Mathematics Subject Classification: 54D20, 54B05, 54B10.

Key words and phrases: selection principles, strongly starcompact, starcompact, starHurewicz, strongly star-Hurewicz. 
In 1925, Hurewicz [8] (see also [9]) introduced the Hurewicz covering property for a space $X$ in the following way:

$\mathrm{H}$ : For each sequence $\left(\mathcal{U}_{n}: n \in \mathbb{N}\right)$ of open covers of $X$ there exists a sequence $\left(\mathcal{V}_{n}: n \in \mathbb{N}\right)$ such that for each $n, \mathcal{V}_{n}$ is a finite subset of $\mathcal{U}_{n}$, and for each $x \in X, x \in \bigcup \mathcal{V}_{n}$ for all but finitely many $n$.

Cammaroto et al. [3] introduced and investigated a generalization of the Hurewicz covering property. Bonanzinga et al. [1] introduced star selection hypotheses similar to the above (see also [10]):

SH: A space $X$ has the star-Hurewicz property (or is a star-Hurewicz space) if for each sequence $\left(\mathcal{U}_{n}: n \in \mathbb{N}\right)$ of open covers of $X$ there exists a sequence $\left(\mathcal{V}_{n}: n \in \mathbb{N}\right)$ such that for each $n, \mathcal{V}_{n}$ is a finite subset of $\mathcal{U}_{n}$, and for each $x \in X, x \in \operatorname{St}\left(\bigcup \mathcal{V}_{n}, \mathcal{U}_{n}\right)$ for all but finitely many $n$.

SSH: A space $X$ has the strongly star-Hurewicz property (or is a Strongly star-Hurewicz space) if for each sequence $\left(\mathcal{U}_{n}: n \in \mathbb{N}\right)$ of open covers of $X$ there exists a sequence $\left(A_{n}: n \in \mathbb{N}\right)$ of finite subsets of $X$ such that for each $x \in X, x \in \operatorname{St}\left(A_{n}, \mathcal{U}_{n}\right)$ for all but finitely many $n$.

From the above definitions, we have the following diagram:

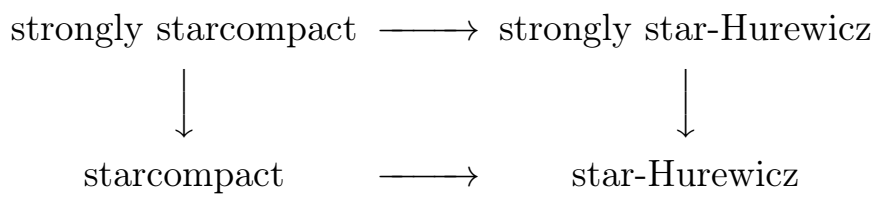

Bonanzinga et al. [1] studied star-Hurewicz and related spaces. The purpose of this paper is to investigate the relationships between star-Hurewicz spaces and related spaces, and also to study topological properties of star-Hurewicz spaces.

Throughout this paper, the extent $e(X)$ of a space $X$ is the smallest infinite cardinal $\kappa$ such that every discrete closed subset of $X$ has cardinality at most $\kappa$. Let $\omega$ denote the first infinite cardinal, $\omega_{1}$ the first uncountable cardinal and $\mathfrak{c}$ the cardinality of the set of all real numbers. For a cardinal $\kappa$, let $\kappa^{+}$be the smallest cardinal greater than $\kappa$. For any ordinals $\alpha, \beta$ with $\alpha<\beta$, we write $[\alpha, \beta),(\alpha, \beta)$ etc. for the usual ordinal intervals. As usual, a cardinal is an initial ordinal and an ordinal is the set of smaller ordinals. Every cardinal is often viewed as a space with the usual order topology. Other terms and symbols that we do not define follow [6].

2. Main results. First we give some examples showing relationships between strongly star-Hurewicz spaces and related spaces. 
EXAMPLE 2.1. There exists a Tychonoff strongly star-Hurewicz (hence star-Hurewicz) space $X$ which is not starcompact (hence not strongly starcompact).

Proof. Consider the subspace

$$
X=([0, \omega] \times[0, \omega]) \backslash\{\langle\omega, \omega\rangle\}
$$

of the product space $[0, \omega] \times[0, \omega]$. Clearly, $X$ is a Tychonoff space.

First we show that $X$ is strongly star-Hurewicz. To this end, let $\left\{\mathcal{U}_{n}\right.$ : $n \in \mathbb{N}\}$ be a sequence of open covers of $X$. For each $n \in \mathbb{N}$, let

$$
K_{n}=([0, \omega] \times[0, n-1]) \cup([0, n-1] \times[0, \omega]) .
$$

Then $K_{n}$ is the union of finite compact subsets of $X$. We can find a finite subset $A_{n}$ of $K_{n}$ such that $K_{n} \subseteq \operatorname{St}\left(A_{n}, \mathcal{U}_{n}\right)$. Note that $\bigcup_{n \in \mathbb{N}} K_{n}=X$. For any $x \in X$, there exists $n_{0} \in \mathbb{N}$ such that $x \in K_{n_{0}}$, thus $x \in \operatorname{St}\left(A_{n}, \mathcal{U}_{n}\right)$ for each $n>n_{0}$, which shows that $X$ is strongly star-Hurewicz.

Next we show that $X$ is not starcompact. For each $n \in \omega$, let

$$
U_{n}=\{n\} \times[0, \omega] \text { and } V_{n}=(n, \omega] \times\{n\} .
$$

Let us consider the open cover

$$
\mathcal{U}=\left\{U_{n}: n \in \omega\right\} \cup\left\{V_{n}: n \in \omega\right\}
$$

of $X$. It suffices to show that $\operatorname{St}(\bigcup \mathcal{V}, \mathcal{U}) \neq X$ for any finite subset $\mathcal{V}$ of $\mathcal{U}$. To see this, let $\mathcal{V}$ be any finite subset of $\mathcal{U}$. There exist $n_{1} \in \omega$ and $n_{2} \in \omega$ such that $U_{n} \notin \mathcal{V}$ for each $n>n_{1}$ and $V_{n} \notin \mathcal{V}$ for each $n>n_{2}$. Pick $n^{\prime}>\max \left\{n_{1}, n_{2}\right\}$; then $V_{n^{\prime}} \cap(\bigcup \mathcal{V})=\emptyset$, hence $\left\langle n^{\prime}, \omega\right\rangle \notin \operatorname{St}(\bigcup \mathcal{V}, \mathcal{U})$, since $V_{n^{\prime}}$ is the only element of $\mathcal{U}$ containing $\left\langle n^{\prime}, \omega\right\rangle$. This shows that $X$ is not starcompact.

EXAMPLE 2.2. There exists a Tychonoff star-Hurewicz space which is not strongly star-Hurewicz.

Proof. Let $D=\left\{d_{\alpha}: \alpha<\mathfrak{c}\right\}$ be a discrete space of cardinality $\mathfrak{c}$ and let $D^{*}=D \cup\left\{d^{*}\right\}$ be one-point compactification of $D$, where $d^{*} \notin D$.

Consider the subspace

$$
X=\left(D^{*} \times\left[0, \mathfrak{c}^{+}\right]\right) \backslash\left\{\left\langle d^{*}, \mathfrak{c}^{+}\right\rangle\right\}
$$

of the product space $D^{*} \times\left[0, \mathfrak{c}^{+}\right]$. Then $X$ is Tychonoff.

To show that $X$ is star-Hurewicz, it suffices to show that $X$ is starcompact, since every starcompact space is star-Hurewicz.

To this end, let $\mathcal{U}$ be an open cover of $X$. For each $\alpha<\mathfrak{c}$, there exists $U_{\alpha} \in \mathcal{U}$ such that $\left\langle d_{\alpha}, \mathfrak{c}^{+}\right\rangle \in U_{\alpha}$, hence we can find $\beta_{\alpha}<\mathfrak{c}^{+}$such that $\left\{d_{\alpha}\right\} \times\left(\beta_{\alpha}, \mathfrak{c}^{+}\right] \subseteq U_{\alpha}$. Let $\beta=\sup \left\{\beta_{\alpha}: \alpha<\mathfrak{c}\right\}$. Then $\beta<\mathfrak{c}^{+}$. Let $K=$ $D^{*} \times\{\beta\}$. Then $K$ is compact and $U_{\alpha} \cap K \neq \emptyset$ for each $\alpha<\mathfrak{c}$. Since $\mathcal{U}$ 
covers $K$, there exists a finite subset $\mathcal{U}^{\prime}$ of $\mathcal{U}$ such that $K \subseteq \cup \mathcal{U}^{\prime}$. Thus

$$
D \times\left\{\mathfrak{c}^{+}\right\} \subseteq \operatorname{St}\left(\bigcup \mathcal{U}^{\prime}, \mathcal{U}\right) .
$$

On the other hand, since $D^{*} \times\left[0, \mathfrak{c}^{+}\right)$is countably compact, it is strongly starcompact (see [5, 11]), so we can find a finite subset $\mathcal{U}^{\prime \prime}$ of $\mathcal{U}$ such that

$$
D^{*} \times\left[0, \mathfrak{c}^{+}\right) \subseteq \operatorname{St}\left(\bigcup \mathcal{U}^{\prime \prime}, \mathcal{U}\right) .
$$

If we put $\mathcal{V}=\mathcal{U}^{\prime} \cup \mathcal{U}^{\prime \prime}$, then $\mathcal{V}$ is a finite subset of $\mathcal{U}$ such that $X=\operatorname{St}(\bigcup \mathcal{V}, \mathcal{U})$, which shows that $X$ is starcompact.

Next we show that $X$ is not strongly star-Hurewicz. For each $\alpha<\mathfrak{c}$, let

$$
U_{\alpha}=\left\{d_{\alpha}\right\} \times\left[0, \mathfrak{c}^{+}\right] .
$$

For each $n \in \mathbb{N}$, let

$$
\mathcal{U}_{n}=\left\{U_{\alpha}: \alpha<\mathfrak{c}\right\} \cup\left\{D^{*} \times\left[0, \mathfrak{c}^{+}\right)\right\} .
$$

Then $\mathcal{U}_{n}$ is an open cover of $X$. Let $\left(A_{n}: n \in \mathbb{N}\right)$ be any sequence of finite subsets of $X$. It suffices to show that there exists $x \in X$ such that $x \notin \operatorname{St}\left(A_{n}, \mathcal{U}_{n}\right)$ for all $n \in \mathbb{N}$.

For each $n \in \mathbb{N}$, since $A_{n}$ is finite, there exists $\alpha_{n}<\mathfrak{c}$ such that

$$
A_{n} \cap U_{\alpha}=\emptyset \quad \text { for each } \alpha>\alpha_{n} .
$$

Let $\beta=\sup \left\{\alpha_{n}: n \in \mathbb{N}\right\}$. Then $\beta<\mathfrak{c}$ and

$$
\left(\bigcup_{n \in \mathbb{N}} A_{n}\right) \cap U_{\alpha}=\emptyset \quad \text { for each } \alpha>\beta .
$$

If we pick $\alpha^{\prime}>\beta$, then $\left\langle d_{\alpha^{\prime}}, \mathfrak{c}^{+}\right\rangle \notin \operatorname{St}\left(A_{n}, \mathcal{U}_{n}\right)$ for each $n \in \mathbb{N}$, since $U_{\alpha^{\prime}}$ is the only element of $\mathcal{U}_{n}$ containing $\left\langle d_{\alpha^{\prime}}, \mathfrak{c}^{+}\right\rangle$; this shows that $X$ is not strongly star-Hurewicz.

REMARK 2.1. Example 2.2 also shows that there exists a Tychonoff starcompact space that is not strongly starcompact.

Recall that the Alexandorff duplicate $A(X)$ of a space $X$ is constructed in the following way: The underlying set of $A(X)$ is $X \times\{0,1\}$; each point of $X \times\{1\}$ is isolated and a basic neighborhood of $\langle x, 0\rangle \in X \times\{0\}$ is of the form $(U \times\{0\}) \cup((U \times\{1\}) \backslash\{\langle x, 1\rangle\})$, where $U$ is a neighborhood of $x$ in $X$.

It is well known that a space $X$ is countably compact if and only if so is $A(X)$. In the following, we give two examples to show that this result cannot be generalized to star-Hurewicz spaces.

EXAmple 2.3. There exists a Tychonoff star-Hurewicz space $X$ such that $A(X)$ is not star-Hurewicz.

Proof. Let $X=\left(D^{*} \times\left[0, \mathfrak{c}^{+}\right]\right) \backslash\left\{\left\langle d^{*}, \mathfrak{c}^{+}\right\rangle\right\}$be the space of Example 2.2. Then $X$ is a star-Hurewicz space with $e(X)=\mathfrak{c}$. 
However, $A(X)$ is not star-Hurewicz. In fact, $(D \times\{\mathfrak{c}\}) \times\{1\}$ is an open and closed subset of $X$ with $|(D \times\{\mathfrak{c}\}) \times\{1\}|=\mathfrak{c}$, and each point $\left\langle\left\langle d_{\alpha}, \mathfrak{c}\right\rangle, 1\right\rangle$ is isolated for each $\alpha<\mathfrak{c}$. Hence $A(X)$ is not star-Hurewicz, since every open and closed subset of a star-Hurewicz space is star-Hurewicz, and $(D \times\{\mathfrak{c}\})$ $\times\{1\}$ is not star-Hurewicz.

To show the next result, we need a lemma from [4].

Lemma 2.4. For a $T_{1}$-space $X, e(X)=e(A(X))$.

From the proof of Example 2.3, it is not difficult to deduce the following result.

TheOREm 2.5. If $X$ is a $T_{1}$-space and $A(X)$ is a star-Hurewicz space, then $e(A(X))<\omega_{1}$.

Proof. By Lemma 2.4, we need to show that $e(X)<\omega_{1}$. Suppose that $e(X) \geq \omega_{1}$. Then there exists a discrete closed subset $B$ of $X$ such that $|B| \geq \omega_{1}$. Hence $B \times\{1\}$ is an open and closed subset of $A(X)$ and every point of $B \times\{1\}$ is isolated. Thus $A(X)$ is not star-Hurewicz, since every open and closed subset of a star-Hurewicz space is star-Hurewicz, and $B \times\{1\}$ is not star-Hurewicz.

REMARK 2.2. The author does not know if the Alexandorff duplicate $A(X)$ of a star-Hurewicz $T_{1}$-space $X$ with $e(X)<\omega_{1}$ is star-Hurewicz.

It is not difficult to prove the following result.

THEOREM 2.6. Every continuous image of a star-Hurewicz space is starHurewicz.

Proof. Let $f: X \rightarrow Y$ be a continuous mapping from a star-Hurewicz space $X$ onto a space $Y$. Let $\left(\mathcal{U}_{n}: n \in \mathbb{N}\right)$ be a sequence of open covers of $Y$. For each $n \in \mathbb{N}$, let $\mathcal{V}_{n}=\left\{f^{-1}(U): U \in \mathcal{U}_{n}\right\}$. It is an open cover of $X$. Since $X$ is star-Hurewicz, there exists a sequence $\left(\mathcal{V}_{n}^{\prime}: n \in \mathbb{N}\right)$ such that for each $n, \mathcal{V}_{n}^{\prime}$ is a finite subset of $\mathcal{U}_{n}$, and for each $x \in X, x \in \operatorname{St}\left(\bigcup \mathcal{V}_{n}^{\prime}, \mathcal{U}_{n}\right)$ for all but finitely many $n$. For each $n \in \mathbb{N}$, let $\mathcal{U}_{n}^{\prime}=\left\{f(U): U \in \mathcal{V}_{n}^{\prime}\right\}$. Then $\mathcal{U}_{n}^{\prime}$ is a finite subset of $\mathcal{U}_{n}$.

We will show that each $y \in Y$ is in $\operatorname{St}\left(\bigcup \mathcal{U}_{n}^{\prime}, \mathcal{U}_{n}\right)$ for all but finitely many $n$. In fact, let $y \in Y$. Then there is $x \in X$ such that $f(x)=y$. Hence $x \in \operatorname{St}\left(\bigcup \mathcal{V}_{n}^{\prime}, \mathcal{V}_{n}\right)$ for all but finitely many $n$. Thus $y=f(x) \in \operatorname{St}(\bigcup\{f(U)$ : $\left.\left.U \in \mathcal{V}_{n}^{\prime}\right\},\left\{f(U): U \in \mathcal{V}_{n}\right\}\right)=\operatorname{St}\left(\bigcup \mathcal{U}_{n}^{\prime}, \mathcal{U}_{n}\right)$ for all but finitely many $n$, which shows that $Y$ is star-Hurewicz.

Next we turn to preimages. We show that the preimage of a star-Hurewicz space under a closed 2-to-1 continuous map need not be star-Hurewicz.

EXAmPle 2.7. There exists a closed 2-to-1 continuous map $f: X \rightarrow Y$ such that $Y$ is star-Hurewicz, but $X$ is not. 
Proof. Just consider the projection map $A(X) \rightarrow X$, where $X$ is the space of Example 2.3.

Now we give a positive result:

THEOREM 2.8. If $f$ is an open perfect continuous map from a space $X$ onto a star-Hurewicz space $Y$, then $X$ is star-Hurewicz.

Proof. Let $\left(\mathcal{U}_{n}: n \in \mathbb{N}\right)$ be a sequence of open covers of $X$ and let $y \in Y$. For each $n \in \mathbb{N}$, since $f^{-1}(y)$ is compact, there exists a finite subcollection $\mathcal{U}_{n_{y}}$ of $\mathcal{U}_{n}$ such that $f^{-1}(y) \subseteq \bigcup \mathcal{U}_{n_{y}}$ and $U \cap f^{-1}(y) \neq \emptyset$ for each $U \in \mathcal{U}_{n_{y}}$. Since $f$ is closed, there exists an open neighborhood $V_{n_{y}}$ of $y$ in $Y$ such that

$$
f^{-1}\left(V_{n_{y}}\right) \subseteq \bigcup\left\{U: U \in \mathcal{U}_{n_{y}}\right\} .
$$

Since $f$ is open, we can assume that

$$
V_{n_{y}} \subseteq \bigcap\left\{f(U): U \in \mathcal{U}_{n_{y}}\right\}
$$

Since $\mathcal{V}_{n}=\left\{V_{n_{y}}: y \in Y\right\}$ is an open cover of $Y$. $Y$ is star-Hurewicz, there exists a sequence $\left(\mathcal{V}_{n}^{\prime}: n \in \mathbb{N}\right)$ such that for each $n \in \mathbb{N}$, $\mathcal{V}_{n}^{\prime}$ is a finite subset of $\mathcal{V}_{n}$, and for each $y \in Y, y \in \operatorname{St}\left(\bigcup \mathcal{V}_{n}^{\prime}, \mathcal{V}_{n}\right)$ for all but finitely many $n$. Without loss of generality, we can assume that $\mathcal{V}_{n}^{\prime}=\left\{V_{n_{y_{i}}}: i<n^{\prime}\right\}$. Let $\mathcal{U}_{n}^{\prime}=\bigcup_{i<n^{\prime}} \mathcal{U}_{n_{y_{i}}}$. Then $\mathcal{U}_{n}^{\prime}$ is a finite subset of $\mathcal{U}_{n}$ and

$$
f^{-1}\left(\bigcup \mathcal{V}_{n}^{\prime}\right) \subseteq \bigcup \mathcal{U}_{n}^{\prime}
$$

Next we show that each $x \in X$ is in $\operatorname{St}\left(\bigcup \mathcal{U}_{n}^{\prime}, \mathcal{U}_{n}\right)$ for all but finitely many $n$. Let $x \in X$. Then $y=f(x) \in \operatorname{St}\left(\bigcup \mathcal{V}_{n}^{\prime}, \mathcal{V}_{n}\right)$ for all but finitely many $n$. For $n \in \mathbb{N}$, if $y=f(x) \in \operatorname{St}\left(\bigcup \mathcal{V}_{n}^{\prime}, \mathcal{V}_{n}\right)$, then there exist $i<n^{\prime}$ and $y^{\prime} \in Y$ such that $V_{n_{y_{i}}} \cap V_{n_{y^{\prime}}} \neq \emptyset$ and $y \in V_{n_{y^{\prime}}}$. Since

$$
x \in f^{-1}\left(V_{n_{y^{\prime}}}\right) \subseteq \bigcup\left\{U: U \in \mathcal{U}_{n_{y^{\prime}}}\right\},
$$

we can choose $U \in \mathcal{U}_{n_{y^{\prime}}}$ with $x \in U$. Then $V_{n_{y^{\prime}}} \subseteq f(U)$ by (1). Hence $U \cap f^{-1}\left(\bigcup \mathcal{V}_{n}^{\prime}\right) \neq \emptyset$. Thus $x \in \operatorname{St}\left(f^{-1}\left(\bigcup \mathcal{V}_{n}^{\prime}\right), \mathcal{U}_{n}\right)$. Therefore $x \in \operatorname{St}\left(\bigcup \mathcal{U}_{n}^{\prime}, \mathcal{U}_{n}\right)$ by (2), which shows that each $x \in X$ is in $\operatorname{St}\left(\bigcup \mathcal{U}_{n}^{\prime}, \mathcal{U}_{n}\right)$ for all but finitely many $n$. Consequently, $X$ is star-Hurewicz.

We have the following corollary from Theorem 2.8.

COROLlary 2.9 ([1]). The product of a star-Hurewicz space and a compact space is star-Hurewicz.

However, the product of two star-Hurewicz spaces need not be starHurewicz. In fact, the following well-known example shows that the product of two countably compact (hence star-Hurewicz) spaces need not be starHurewicz. We sketch the proof for completeness. 
EXAMPLE 2.10. There exist two Tychonoff countably compact (hence star-Hurewicz) spaces $X$ and $Y$ such that $X \times Y$ is not star-Hurewicz.

Proof. Let $D$ be a discrete space of cardinality c. We define $X=\bigcup_{\alpha<\omega_{1}} E_{\alpha}$ and $Y=\bigcup_{\alpha<\omega_{1}} F_{\alpha}$, where $E_{\alpha}$ and $F_{\alpha}$ are subsets of $\beta D$ which are defined inductively so as to satisfy the following conditions:

(1) $E_{\alpha} \cap F_{\beta}=D$ if $\alpha \neq \beta$;

(2) $\left|E_{\alpha}\right| \leq \mathfrak{c}$ and $\left|F_{\beta}\right| \leq \mathfrak{c}$;

(3) every infinite subset of $E_{\alpha}$ (resp., $F_{\alpha}$ ) has an accumulation point in $E_{\alpha+1}$ (resp., $\left.F_{\alpha+1}\right)$.

The sets $E_{\alpha}$ and $F_{\alpha}$ are well-defined since every infinite closed set in $\beta D$ has cardinality $2^{\mathfrak{c}}$ (see [13]). The diagonal $\{\langle d, d\rangle: d \in D\}$ is an open and closed subset of $X \times Y$ with cardinality $\mathfrak{c}$ and $\{\langle d, d\rangle\}$ is isolated for each $d \in D$. Thus $X \times Y$ is not star-Hurewicz, since open and closed subsets of star-Hurewicz spaces are star-Hurewicz, and the diagonal $\{\langle d, d\rangle: d \in D\}$ is not star-Hurewicz.

In [5, Example 3.3.3], van Douwen et al. gave an example of a countably compact (and hence star-Hurewicz) space $X$ and a Lindelöf space $Y$ such that $X \times Y$ is not strongly star-Lindelöf. Now, we shall show that $X \times Y$ is not star-Hurewicz either. We need the following lemma.

LEMMA $2.11([2])$. A space $X$ is a star-Hurewicz space iff for every sequence $\left(\mathcal{U}_{n}: n \in \mathbb{N}\right)$ of open covers of $X$ there exists a finite $\mathcal{V}_{n} \subseteq \mathcal{U}_{n}(n \in \mathbb{N})$ such that for every $x \in X, \operatorname{St}\left(x, \mathcal{U}_{n}\right) \cap\left(\bigcup \mathcal{V}_{n}\right) \neq \emptyset$ for all but finitely many $n \in \mathbb{N}$.

EXAMPLE 2.12. There exist a countably compact (hence star-Hurewicz) space $X$ and a Lindelöf space $Y$ such that $X \times Y$ is not star-Hurewicz.

Proof. Let $X=\left[0, \omega_{1}\right)$ with the usual order topology and $Y=\left[0, \omega_{1}\right]$ with the following topology: each point $\alpha$ with $\alpha<\omega_{1}$ is isolated and a set $U$ containing $\omega_{1}$ is open if and only if $Y \backslash U$ is countable. Then $X$ is countably compact and $Y$ is Lindelöf.

Now, we show that $X \times Y$ is not star-Hurewicz. For each $\alpha<\omega_{1}$, let

$$
U_{\alpha}=[0, \alpha] \times\left[\alpha, \omega_{1}\right] \text { and } V_{\alpha}=\left(\alpha, \omega_{1}\right) \times\{\alpha\} .
$$

Then

$$
U_{\alpha} \cap V_{\alpha^{\prime}}=\emptyset \quad \text { for any } \alpha<\omega_{1} \text { and } \alpha^{\prime}<\omega_{1}
$$

and

$$
V_{\alpha} \cap V_{\alpha^{\prime}}=\emptyset \quad \text { if } \alpha \neq \alpha^{\prime}
$$

For each $n \in \mathbb{N}$, let

$$
\mathcal{U}_{n}=\left\{U_{\alpha}: \alpha<\omega_{1}\right\} \cup\left\{V_{\alpha}: \alpha<\omega_{1}\right\} .
$$


Then $\mathcal{U}_{n}$ is an open cover of $X \times Y$. By Lemma 2.11 it suffices to show that for any sequence $\left(\mathcal{V}_{n}: n \in \mathbb{N}\right)$ of finite sets such that $\mathcal{V}_{n} \subseteq \mathcal{U}_{n}$ for each $n \in \mathbb{N}$, there exists a point $a \in X \times Y$ such that $\operatorname{St}\left(a, \mathcal{U}_{n}\right) \cap\left(\bigcup \mathcal{V}_{n}\right)=\emptyset$ for all $n \in \mathbb{N}$.

Let $\left(\mathcal{V}_{n}: n \in \mathbb{N}\right)$ be any sequence such that for each $n \in \mathbb{N}, \mathcal{V}_{n}$ is a finite subset of $\mathcal{U}_{n}$. For each $n \in \mathbb{N}$, since $\mathcal{V}_{n}$ is finite, there exists $\alpha_{n}<\omega_{1}$ such that

$$
V_{\alpha} \notin \mathcal{V}_{n} \quad \text { for each } \alpha>\alpha_{n} .
$$

Let $\beta=\sup \left\{\alpha_{n}: n \in \mathbb{N}\right\}$. Clearly, $\beta<\omega_{1}$. Thus we have

$$
V_{\alpha} \notin \mathcal{V}_{n} \quad \text { for each } \alpha>\beta \text { and } n \in \mathbb{N} \text {. }
$$

Pick $\alpha>\beta$. Since $V_{\alpha}$ is the only element of $\mathcal{U}_{n}$ containing the point $\langle\alpha+1, \alpha\rangle$, and $V_{\alpha} \cap V=\emptyset$ for each $V \in \mathcal{V}_{n}$ and $n \in \mathbb{N}$, it follows that $\operatorname{St}\left(\langle\alpha+1, \alpha\rangle, \mathcal{U}_{n}\right)$ $=V_{\alpha}$ and $V_{\alpha} \cap\left(\bigcup \mathcal{V}_{n}\right)=\emptyset$ for $n \in \mathbb{N}$.

Next we give a condition under which paraLindelöfness implies Lindelöfness. Recall that a space $X$ is paraLindelöf if every open cover $\mathcal{U}$ of $X$ has a locally countable open refinement.

THEOREM 2.13. Every paraLindelöf star-Hurewicz space is Lindelöf.

Proof. Let $X$ be a paraLindelöf star-Hurewicz space and $\mathcal{U}$ be an open cover of $X$. Then there exists a locally countable open refinement $\mathcal{V}$ of $\mathcal{U}$. For each $x \in X$, there exists an open neighborhood $V_{x}$ of $x$ such that $V_{x} \subseteq V$ for some $V \in \mathcal{V}$ and $\left\{V \in \mathcal{V}: V_{x} \cap V \neq \emptyset\right\}$ is countable. Let $\mathcal{V}^{\prime}=\left\{V_{x}: x \in X\right\}$. Then $\mathcal{V}^{\prime}$ is an open refinement of $\mathcal{V}$. Since $X$ is star-Hurewicz, there exists a sequence $\left(\mathcal{V}_{n}^{\prime}: n \in \mathbb{N}\right)$ such that for each $n, \mathcal{V}_{n}^{\prime}$ is a finite subset of $\mathcal{V}^{\prime}$, and for each $x \in X, x \in \operatorname{St}\left(\bigcup \mathcal{V}_{n}^{\prime}, \mathcal{V}^{\prime}\right)$ for all but finitely many $n$.

For each $n \in \mathbb{N}$, let

$$
\mathcal{V}_{n}=\left\{V \in \mathcal{V} \text { : there exists some } V^{\prime} \in \mathcal{V}_{n}^{\prime} \text { such that } V \cap V^{\prime} \neq \emptyset\right\} \text {. }
$$

Then $\mathcal{V}_{n}$ is a countable subset of $\mathcal{V}$. Let $\mathcal{W}=\bigcup_{n \in \mathbb{N}} \mathcal{V}_{n}$. Then $\mathcal{W}$ is a countable open cover of $X$. For each $V \in \mathcal{W}$, choose $U_{V} \in \mathcal{U}$ such that $V \subseteq U_{V}$. Then $\left\{U_{V}: V \in \mathcal{W}\right\}$ is a countable subcover of $\mathcal{U}$, which shows that $X$ is Lindelöf.

Since every paracompact space is paraLindelöf, the following corollary follows from Theorem 2.13.

Corollary 2.14. A paracompact star-Hurewicz space $X$ is Lindelöf.

Acknowledgments. The author acknowledges the support from the National Natural Science Foundation of China (grant 11271036). 


\section{References}

[1] M. Bonanzinga, F. Cammaroto and Lj. D. R. Kočinac, Star-Hurewicz and related spaces, Appl. Gen. Topology 5 (2004), 79-89.

[2] M. Bonanzinga, F. Cammaroto, Lj. D. R. Kočinac and M. V. Matveev, On weaker forms of Menger, Rothberger and Hurewicz properties, Mat. Vesnik 61 (2009), 13-23.

[3] F. Cammaroto, Lj. D. R. Kočinac and G. Nordo, $\mathcal{F}$-Hurewicz spaces, Questions Answers Gen. Topology 22 (2004), 23-32.

[4] J.-L. Cao and Y.-K. Song, Aquaro number versus absolute star-Lindelöf number, Houston J. Math. 29 (2003), 925-936.

[5] E van Douwen, G. K. Reed, A. W. Roscoe and I. J. Tree, Star covering properties, Topology Appl. 39 (1991), 71-103.

[6] R. Engelking, General Topology, Heldermann, Berlin, 1989.

[7] W. M. Fleischman, A new extension of countable compactness, Fund. Math. 67 (1971), 1-7.

[8] W. Hurewicz, Über eine Verallgemeinerung des Borelschen Theorems, Math. Z. 24 (1925), 401-421.

[9] W. Hurewicz, Über Folgen stetiger Funktionen, Fund. Math. 8 (1927), 193-204.

[10] Lj. D. R. Kočinac, Selected results on selection principles, in: Proceedings of the 3rd Seminar on Geometry and Topology, Sh. Rezapour (ed.), Tabriz, 2004, 71-104.

[11] M. V. Matveev, A survey on star-covering properties, Topology Atlas, preprint no. 330, 1998.

[12] Y.-K. Song, On countable star-covering spaces, Appl. Gen. Topology 8 (2007), 249258.

[13] R. C. Walker, The Stone-Čech Compactification, Springer, Berlin, 1974.

Yan-Kui Song

Institute of Mathematics

School of Mathematical Sciences

Nanjing Normal University

Nanjing 210023, P.R. China

E-mail: songyankui@njnu.edu.cn 
